\title{
An economic model of amniocentesis choice
}

\author{
Eduardo Fajnzylbera, V. Joseph Hotz ${ }^{b,}{ }^{*}$, and Seth G. Sanders ${ }^{c}$ \\ aSchool of Government, Universidad Adolfo Ibáñez, Peñalolén, Chile \\ bDepartment of Economics and Duke Population Research Institute, Duke University, Durham, \\ NC 27705, U.S.A. \\ 'Department of Economics, Sanford School of Public Policy and Duke Population Research \\ Institute, Duke University, Durham, NC 27705, U.S.A.
}

\section{Abstract}

\begin{abstract}
Medical practitioners typically utilize the following protocol when advising pregnant women about testing for the possibility of genetic disorders with their fetus: Pregnant women over the age of 35 should be tested for Down syndrome and other genetic disorders, while for younger women, such tests are discouraged (or not discussed) as the test can cause a pregnancy to miscarry. The logic appears compelling. The rate at which amniocentesis causes a pregnancy to miscarry is constant while the rate of genetic disorder rises substantially over a woman's reproductive years. Hence the potential benefit from testing - being able to terminate a fetus that is known to have a genetic disorder - rises with maternal age. This article argues that this logic is incomplete. While the benefits to testing do rise with age, the costs rise as well. Undergoing an amniocentesis always entails the risk of inducing a miscarriage of a healthy fetus. However, these costs are lower at early ages, because there is a higher probability of being able to replace a miscarried fetus with a healthy birth at a later age. We develop and calibrate a dynamic model of amniocentesis choice to explore this tradeoff. For parameters that characterize realistic age patterns of chromosomal abnormalities, fertility rates and miscarriages following amniocentesis, our model implies a falling, rather than rising, rate of amniocentesis as women approach menopause.
\end{abstract}

\section{Introduction}

Amniocentesis and chorionic villus sampling (CVS) are methods of diagnosing Downs Syndrome and other genetic disorders. Amniocentesis and transabdominal CVS involve inserting a thin hollow needle through a pregnant woman's abdomen to remove fetal cells from the amniotic sac (amniocentesis) or from around the chorionic villi (CVS). ${ }^{1}$ Microscopic examination of the chromosome size and banding patterns of these fetal cells allows medical laboratories to identify and arrange each of the 24 different chromosomes (22 pairs of autosomes and one pair of sex chromosomes) (a karyotype), which then serves

\footnotetext{
(C) No copyright information found. Please enter mannually.

*Corresponding author: Tel. 919-660-1841; fax: 919-684-8974. NOTE: Between September 1, 2010 and May 30, 2011, send all correspondence to, V. Joseph Hotz, The Russell Sage Foundation, 112 East 64th Street, New York, NY 10065, Tel: 1 (212) 750-6000, Fax: 1 (212) 371-4761.

Publisher's Disclaimer: This is a PDF file of an unedited manuscript that has been accepted for publication. As a service to our customers we are providing this early version of the manuscript. The manuscript will undergo copyediting, typesetting, and review of the resulting proof before it is published in its final citable form. Please note that during the production process errors may be discovered which could affect the content, and all legal disclaimers that apply to the journal pertain.

${ }^{1}$ Transcervical CVS involves inserting a thin tube through a woman's vagina and cervix to the villi, and using suction to remove a small sample of fetal cells. The risks involved in transcervical and transabdominal CVS are the same and equally prevalent. For a concise discussion of CVS methods and risks, see http://www.modimes.org/HealthLibrary2/factsheets/ChorionicVillusSampling.htm
} 
as a tool in the diagnosis of genetic diseases. An extra copy of chromosome 21 in a karyotype identifies Down syndrome the most common genetic disorder (National Center for Health Statistics, 1994).

Children with Down syndrome suffer from general hypotonia (poor muscle strength and elasticity), mental retardation, growth retardation, and are at significant risk for congenital malformations of which heart problems are the most common. While some therapies are available for specific malformations there are no proven therapies available to treat the cognitive problems associated with Down syndrome (Dick, et al., 1996). In developing countries, life expectancy for children with Down syndrome is approximately 35 years (Brazil) but advances in medical treatment for specific conditions has increased lifeexpectancy in the U.S. to approximately 55 years. ${ }^{2}$ The lifetime economic cost of Down syndrome is estimated at $\$ 410,000$ per child based on U.S. cross-sectional data from 1988 (Waitzman, Romano \& Scheffler, 1994). This of course greatly underestimates the full cost to families which include maternal depression and difficulties with marital and sibling relationships (Dick, et al., 1996). Down syndrome accounts for about half of chromosomal abnormalities detected by karyotyping. Spina bifida (an incompletely enclosed spinal cord) and anencephaly (the absence of a large part of the brain and skull) are two less common but more fatal genetic disorders detected by amniocentesis and CVS.

Both CVS and amniocentesis provide very accurate diagnosis of Down syndrome in a fetus, greater than $99 \%$. However, both have important risks that a pregnant woman must consider. The amniocentesis procedure results in a spontaneous miscarriage for 1 in 100 to 1 in 200 women and the CVS procedure carries an even greater risk of procedure-related fetal loss (between $1 \%$ and $1.5 \%$ ). ${ }^{3}$ And while the fraction of pregnancies afflicted with Down syndrome and other genetic disorders rises with maternal age, even at age 49 the vast majority of pregnancies will prove to be chromosomally normal. What this means is that when amniocentesis induces the loss of a fetus, the mother almost always looses a fetus that would have developed into a healthy child.

The current standard in obstetric practice is to recommend that women pregnant at age 35 or older have an amniocentesis or CVS but this is not recommended for pregnant women prior to that age. The American College of Obstetricians and Gynecologists, the College of Medical Genetics and the Canadian Task Force on Periodic Health Examination all agree that a woman who is pregnant and is 35 years old or older should be tested using either amniocentesis or CVS. The recommendations for women who are pregnant and younger than age 35 varies, but generally either the "triple marker" test or no test at all is recommended. ${ }^{4}$ Using age 35 as an age to start testing is based on a compelling, but we argue, incomplete logic. Figure 1 graphs the estimated number of fetuses afflicted with a chromosomal abnormality per 100,000 pregnancies by maternal age. Figure 1 also displays an upper and lower bound estimate on the number of fetuses lost for every 100,000 amniocenteses performed. It is clear that prior to ages 35-38 more healthy fetuses would be lost from performing amniocentesis on all women than would be the number of chromosomally abnormal fetuses detected. It is further argued that, after age 35-38, when chromosomal abnormalities rise rapidly, the rate of successful detection of rate

\footnotetext{
${ }^{2}$ For life expectancy for those born with Down syndrome in Brazil see http://www.epub.org.br/cm/n04/doenca/down/down_i.htm and for the U.S. see http://www.ndss.org/aboutds/aboutds.html.

${ }^{3}$ See U.S. Preventative Service Task Force. (1996). The CVS procedure is feasible to perform earlier in a pregnancy than is amniocentesis.

${ }^{4}$ The "triple marker" test is a screen of a pregnant woman's blood for particular proteins and is an alternative to amniocentesis and CVS. While this test carries lower medical risks, it is far less accurate and typically it detects only $60 \%$ of Down syndrome fetuses. Some doctors will perform amniocentesis on women who test positive on the "triple marker" test. Because of the extremely high false positive rate others will not use the test at all.
} 
chromosomal abnormal fetuses via amniocenteses exceeds the incidence of miscarriages resulting from administering this procedure. It is the latter claim (and accompanying evidence) that has led the medical profession to adopt age 35 as the threshold age for recommending genetic testing to patients.

Economists have criticized this threshold-age approach. For example, Sicherman, Bombard and Rappoport (1995) (SBR hereafter) point out that this approach makes sense from an expected utility theory viewpoint only if the utility loss from a miscarriage due to having an amniocentesis equals the utility gain from avoiding bearing and raising a Down syndrome child. Clearly, women (parents) may differ with respect to how they value the "pain and loss" associated with miscarrying a fetus, on terminating a pregnancy through abortion, and on how they value having additional children, including the relative utility gained from a healthy child versus one born with a chromosomal abnormality. SBR argue that a woman's individual costs and benefits of each outcome can be combined with the probabilities of those outcomes to better aid women in their choice.

Like SBR, this paper relies on an expected utility approach but differs from SBR in an important way. While SBR set the expected utility model in a static context, our paper concentrates on the dynamic decisions inherent in family planning. Specifically, we model a woman's choice about amniocentesis for a particular pregnancy within her lifetime choices over the quantity and quality of children.

There is a long tradition in demography that recognizes the importance of the outcomes for one child or one pregnancy on future choices about childbearing. For example, demographers have extensively studied changes in fertility in response to infant mortality, especially in developing countries (Shultz, 1969, 1976; Ben-Porath, 1976; Preston, 1978; Sah, 1991; Wolpin, 1997). In our model, a procedure-induced miscarriage is viewed as in utero "infant mortality" but women can partially control this risk by their decisions of whether or not to have an amniocentesis. In particular, by not undergoing an amniocentesis, a woman can limit the risk of this form of infant mortality. At the same time, by not having an amniocentesis, she increases her chances of bearing a child with Down syndrome or another genetic disorder. In making her genetic-testing choice, a woman is always cognizant that she can replace a miscarried fetus, with some probability, at later ages. However, this option decreases as a woman approaches menopause. As a result, the "cost" of an amniocentesis rises with maternal age. This is the key different between our model and static expected utility models. In particular, the static model of SBR implies that women will always have a rising propensity to choose to undergo an amniocentesis because of her desire to avoid having a child born with a genetic disorder. In our dynamic model, this propensity may or may not rise with maternal age, precisely because women must balance the benefits associated with having an amniocentesis (avoiding having a child with a genetic disorder) with the costs of such procedures (running the risk of a procedure-induced miscarriage of a healthy fetus).

The remainder of this article is organized as follows. Section 2 lays out a dynamic model of amniocentesis. We show that if the rate of Down syndrome were constant over maternal age, it would be optimal, in the sense of maximizing expected utility, for women to elect an amniocentesis at younger ages rather than at older ages. Since genetic disorders do rise with maternal age, this result makes clear that how the optimal choice changes with maternal age will depend on the rate of increase in genetic disorders relative to the rate of decrease in the probability of replacing a child lost by a procedure-induced amniocentesis. After laying out how the rates of amniocentesis vary by age and birth parity in U.S. data in Section 3, we then solve more realistic models, using numerical methods and plausible parameter values, to determine what the age pattern of amniocentesis choice might look like under reasonable 
biological parameters. The results of these simulations are presented in Section 4. Some implications of our simple model are strongly rejected by the data. For example, the data shows a clear jump in amniocentesis rates at age 35, the age threshold used by medical practitioners. However, other implications of the model are consistent with the data in Section 3, including: (a) a decline in amniocentesis rates as women approach the end of their reproductive years, (b) a higher rate of amniocentesis for lower parity children at most maternal ages, and (c) a larger drop in amniocentesis rates for lower parity children as a woman reaches menopause. Section 5 provides concluding observations.

\section{Modeling Amniocentesis Choice}

\subsection{The Elements of a Dynamic Model}

Consider a model in which women, at each age $t, t=1, \ldots, T$, become pregnant. We treat pregnancies as biologically (and exogenously) determined, occurring with probability $p_{t}, 0<$ $p_{t}<1$. Age $T+1$ is the age of menopause, after which woman's probability of conception is zero (i.e., $p_{T+1} \equiv 0$ ). Pregnant women confront three sequential choices at each age at which they are fecund. First, a pregnant woman may choose to abort the fetus, prior to any genetic testing. Let this choice be denoted by $C_{t}^{A}$, where $C_{t}^{A}$ is either equal to Abort or $\sim$ Abort. Second, conditional on not choosing to initially abort her pregnancy, a woman chooses whether or not to have a test, $\tau$, for a genetic disorder, i.e., an amniocentesis. Let this choice be denoted by $C_{t}^{\tau / \sim A}$, where $C_{t}^{\tau \mid \sim A}$ is either equal to Amnio or $\sim$ Amnio. We assume that the outcome on an amniocentesis $(\tau=+$ or - ) is always accurate. Third, conditional on not initially aborting and having a positive test, women choose whether or not to abort the fetus. Let $C_{t}^{(A \mid \tau=+\& \sim A)}$ denote this choice, where $C_{t}^{(A \mid \tau=+\& \sim A)}$ is either equal to Abort or $\sim A b o r t$. Those women who do choose to have an amniocentesis, $C_{t}^{\tau \mid \sim A}=$ Amnio, may experience a (spontaneous) miscarriage, with probability $m, 0<m<1$. If the mother chooses not to undergo an amniocentesis, the pregnancy will end in a normal birth with probability $q$ and a birth with a genetic disorder with probability $(1-q)$. If she undergoes the procedure, the result of the test will be negative (no genetic abnormalities) or positive with the same probabilities $(q$ and $1-q)$. The probability of a genetic disorder, $(1-q)$, varies with the woman's age.

The above set of actions and probabilistically-occurring events result in a set of potential outcomes at each age. In particular, women may have: a normal birth at age $t$, denoted by $n_{t}$ $=1$; a birth with a genetic disorder (Down syndrome), denoted by $d_{t}=1$; a miscarriage, denoted by $m_{t}=1$; an abortion, denoted by $a_{t}=1$; or no pregnancy, which is (implicitly) recorded by $n_{t}=d_{t}=m_{t}=a_{t}=0$. Let the cumulative values, or "stocks," of these outcomes as of age $t$ be denoted as $X_{t}=X_{t-1}+x_{t}$, where $X=N, D, M$, and $A$ correspond to the outcomes $x=n, d, m$, and $a$.

An essential feature of our model is that children, regardless of their type, are enduring goods. Furthermore, a couple's other choices may have long run consequences. As such, we assume that parent's instantaneous utility, $u_{t}$, is a function of the accumulated choices and outcomes. Furthermore, couples will differ on the utility value of each of these. For example, some couples may get as much utility from a Down child as a healthy child, and other couples (women) may face a large utility cost of abortion while other women face none. These differences in tastes are known to a couple but are unknown to the econometrician. We model the instantaneous utility function as the sum of the utilities derived over the accumulation of each pregnancy event. That is, ${ }^{5}$ 


$$
u_{t}=\sum_{i=1}^{D_{t}} \gamma_{i}^{D}+\sum_{i=1}^{N_{t}} \gamma_{i}^{N}+\sum_{i=1}^{M_{t}} \mu_{i}+\sum_{i=1}^{A_{t}} \alpha_{i}
$$

where $\alpha_{i}=$ Present Discounted Disutility of the $i^{\text {th }}$ abortion; $\gamma_{i}^{D}=$ Present Discounted Utility of the $i^{\text {th }}$ Down child; $\gamma_{i}^{N}=$ Present Discounted Utility of the $i^{\text {th }}$ healthy child; and $\mu_{i}=$ Present Discounted Disutility of the $i^{\text {th }}$ miscarriage. For example, the first healthy child will bring utility $\gamma_{1}^{N}$ for every age after conception. Even though a woman can only conceive between $t=1$ and $t=T$, we assume that a woman will enjoy the utility from a child forever. 6

We assume a couple (woman) will make their (her) decisions between $t=1$ and $t=T$, looking to maximize their (her) expected lifetime utility function:

$$
\max _{\left\{\left(C_{s}^{A}, C_{s}^{\tau \mid A A}, C_{s}^{A \mid \tau=+\& \sim A}\right)\right\}_{s=1}^{T}} U=\sum_{s=1}^{T} \beta^{s-1} E_{s}\left[u_{s}\right] .
$$

The additive separability of the utility function allows us to express the dynamic programming problem in terms of the Present Discounted Utility (PDU) that will be enjoyed over the lifetime from a particular event. That way, at each age, a woman's contemporaneous utility is defined by the decisions she takes (which affect the probabilities of the different outcomes) and the PDU associated with each outcome (which depend on the state variables, $D_{t}, N_{t}, M_{t}$ and $A_{t}$ ).

The dynamic programming problem can then be written as

$$
V_{t}\left(D_{t}, N_{t}, M_{t}, A_{t}\right)=\max _{\left(C_{t}^{A}, C_{t}^{\tau \mid \sim A}, C_{t}^{A \mid \tau=+\& \sim A}\right)}\left\{\begin{array}{l}
p\left[\alpha_{A_{t}+1}+\beta V_{t+1}\left(D_{t}, N_{t}, M_{t}, A_{t}+1\right)\right] \operatorname{Pr}\left(a_{t}=1 \mid C_{t}^{A}, C_{t}^{\tau \mid \sim A}, C_{t}^{A \mid \tau=+\& \sim A}\right) \\
+p\left[\gamma_{D_{t+1}}^{D}+\beta V_{t+1}\left(D_{t}+1, N_{t}, M_{t}, A_{t}\right)\right] \operatorname{Pr}\left(d_{t}=1 \mid C_{t}^{A}, C_{t}^{\tau \mid \sim A}, C_{t}^{A \mid \tau=+\& \sim A}\right) \\
+p\left[\gamma_{N_{t+1}}^{N}+\beta V_{t+1}\left(D_{t}, N_{t}+1, M_{t}, A_{t}\right)\right] \operatorname{Pr}\left(n_{t}=1 \mid C_{t}^{A}, C_{t}^{\tau \mid \sim A}, C_{t}^{A \mid \tau=+\& \sim A}\right) \\
+p\left[\mu_{M_{t}+1}+\beta V_{t+1}\left(D_{t}, N_{t}, M_{t}+1, A_{t}\right)\right] \operatorname{Pr}\left(m_{t}=1 \mid C_{t}^{A}, C_{t}^{\tau \mid \sim A}, C_{t}^{A \mid \tau=+\& \sim A}\right) \\
+(1-p) \beta V_{t+1}\left(D_{t}, N_{t}, M_{t}, A_{t}\right)
\end{array}\right\},
$$

and the choice and outcome probabilities are given by

$$
\operatorname{Pr}\left(a_{t}=1 \mid C_{t}^{A}, C_{t}^{\tau \mid \sim A}, C_{t}^{A \mid \tau=+\& \sim A}\right)=1\left\{C_{t}^{A}=A b o r t\right\}+1\left\{C_{t}^{A}=\sim A b o r t\right\} \cdot 1\left\{C_{t}^{\tau \mid \sim A}=\text { Amnio }\right\}\left(1-m_{t}\right)\left(1-q_{t}\right) \cdot 1\left\{C_{t}^{A \mid \tau=+\& \sim A}=A b o r t\right\}
$$

\footnotetext{
5 This specification of the utility function is quite general. We do not require that gains and losses have a symmetric effect on parents' (mother's) utility. In particular, our specification can allow for forms of loss aversion.

6 More generally, we only require that all fertility-related events of the same type (healthy, Down, abortion or miscarriage) generate a flow of utility for the same number of periods, regardless of the age at which they were experienced. For example, children can be enjoyed for many years, whereas the effect of abortions and miscarriages could last for only a few periods.
} 


$$
\begin{aligned}
\operatorname{Pr}\left(d_{t}=\right. & \left.1 \mid C_{t}^{A}, C_{t}^{\tau \mid \sim A}, C_{t}^{A \mid \tau=+\& \sim A}\right) \\
& =1\left\{C_{t}^{A}=\sim A \text { bort }\right\}\left[1\left\{C_{t}^{\tau \mid \sim A}=\sim \text { Amnio }\right\}\left(1-q_{t}\right)+1\left\{C_{t}^{\tau \mid \sim A}=\text { Amnio }\right\}\left(1-m_{t}\right)\left(1-q_{t}\right) \cdot 1\left\{C_{t}^{A \mid \tau=+\& \sim A}=\sim A \text { bort }\right\}\right]
\end{aligned}
$$

$$
\begin{gathered}
\operatorname{Pr}\left(n_{t}=1 \mid C_{t}^{A}, C_{t}^{\tau \mid \sim A}, C_{t}^{A \mid \tau=+\& \sim A}\right)=1\left\{C_{t}^{A}=\sim A \text { bort }\right\}\left[1\left\{C_{t}^{\tau \mid \sim A}=\sim \text { Amnio }\right\} q_{t}+1\left\{C_{t}^{\tau \mid \sim A}=\text { Amnio }\right\}\left(1-m_{t}\right) q_{t}\right] \\
\operatorname{Pr}\left(m_{t}=1 \mid C_{t}^{A}, C_{t}^{\tau \mid \sim A}, C_{t}^{A \mid \tau=+\& \sim A}\right)=1\left\{C_{t}^{A}=\sim A \text { Abort }\right\} \cdot 1\left\{C_{t}^{\tau \mid \sim A}=\text { Amnio }\right\} m_{t},
\end{gathered}
$$

where the operator, $1\{x\}$, is equal to one if decision $x$ is taken and 0 otherwise. (The structure of the woman's decision problem at age $t$ is illustrated as a decision tree in Figure 2.) Notice that the probabilities are age dependent because some of the parameters, like the probability of having a child with Down syndrome $\left(q_{t}\right)$, are age dependent. We assume that $\gamma_{i}^{D}<\gamma_{i}^{N}$, for all births, i.e., having a healthy child is always preferred to having a child with Down syndrome, and that $\mu_{i} \leq 0$ and $\alpha_{i} \leq 0$, for all births, i.e., abortions and miscarriages are never utility-enhancing.

\subsection{A Simplified Version of the Model}

In this section, we consider a simplified version of this more general dynamic model to illustrate the sorts of implications that it can generate. Assume, for now, that a woman desires only one child. Assume that $\gamma_{1}^{N}=1, \gamma_{1}^{D} \leq 1$, and $\mu_{1}=0, \alpha_{1}=0$, i.e., an abortion or a miscarriage have no utility loss, a normal birth is normalized to a utility value of 1 and less utility is derived from Down syndrome child than a healthy child (but utility could be positive or negative). It follows that $u_{t}\left(D_{t}=0, N_{t}=0, M_{t}, A_{t}\right)=0$ prior to a woman's first birth. At the time of a first birth, a woman's utility is either $u_{t}\left(D_{t}=0, N_{t}=1, M_{t}, A_{t}\right)=1$, if the child is healthy, or $u_{t}\left(D_{t}=1, N_{t}=0, M_{t}, A_{t}\right)=\gamma_{1}^{D} \leq 1$, if the child is born with Down syndrome. As the woman desires only one child, we can assume that after the birth of her child a woman receives her discounted utility payoff and then dies. If she does not have a child by age $T$ (the age of menopause), her utility is zero. Finally, for now, we assume that $m, q$, and $p$ do not vary with maternal age. While many of these simplifying assumptions are either unrealistic or factually inaccurate, they allow us to simplify a woman's fertility decision-making problem. For example, it follows immediately from the above assumptions that in our simplified model we can abstract from the choice of whether to abort a pregnancy prior to genetic testing $\left(C_{t}^{A}=A b o r t\right){ }^{7}$ We consider the two remaining choices and describe how this simplified model can be solved recursively.

Last Stage, Age T-Consider the last stage of the woman's decision problem, at age $T$, after which she can no longer become pregnant. Figure 3 displays the decision tree for this age. We first consider the abortion decision, given a positive outcome on anniocentesis $\left(C_{t}^{A \mid \tau=+\& \sim A}\right)$ and then determine her optimal amniocentesis decision rule.

\footnotetext{
${ }^{7}$ Since the choice to abort after genetic testing is available, and since abortion carries no disutility, a woman will never abort prior to genetic testing if $\gamma_{i}^{N}>0$.
} 
If a woman has had an amniocentesis, avoided a procedure-induced miscarriage and then tested positive for Down syndrome, the woman will abort the pregnancy if the value of a Down syndrome child is negative, since the only issue is whether a child afflicted with Down syndrome will produce positive utility to the woman. Thus, it follows that the optimal abortion choice at age $T$ is characterized by the following decision rule:

$$
C_{T}^{A \mid \tau=+\& \sim A}=\left\{\begin{array}{l}
A b o r t \text { if } \gamma_{1}^{D}<0 \\
\sim A b o r t \text { if } \gamma_{1}^{D} \geq 0
\end{array} .\right.
$$

Given a value of $\gamma_{1}^{D}$, a woman will know whether she will abort should she be faced with a fetus with a genetic disorder and can make a choice at age $T$ on whether to undergo amniocentesis. If $\gamma_{1}^{D} \geq 0$, we know that the woman will not abort. We also know she will not undergo an amniocentesis; doing so generates no present benefits (she will not abort if she tests positive) and entails the risk of miscarriage. In contrast, a woman with $\gamma_{1}^{D}<0$ would abort a fetus if it tested positive for Down syndrome. It follows that the woman's her expected utility is

$$
E\left(u_{T} \mid \text { pregnant }, C_{T}^{\tau}=\text { Amnio, } C_{T}^{A \mid \tau=+\& \sim A}=A b o r t\right)=(1-m) q
$$

if she undergoes an amniocentesis and

$$
E\left(u_{T} \mid \text { pregnant }, C_{T}^{\tau}=\sim \text { Amnio }\right)=q+(1-q) \gamma_{1}^{D}
$$

otherwise. Equating (8) and (9) and solving for $\gamma$ yields

$$
\gamma_{T}^{*}=-m\left(\frac{q}{1-q}\right)
$$

which is that level of utility, as of age $T$, at which a woman is indifferent between having an amniocentesis and aborting if it tests positive and not having an amniocentesis. Thus, $\gamma_{T}^{*}$ is the critical (utility) value that characterizes the optimal decision rule for a woman's amniocentesis choice at age $T$ :

$$
C_{T}^{\tau \mid \sim A}=\left\{\begin{array}{l}
\text { Amnio if } \gamma_{1}^{D}<\gamma_{T}^{*} \\
\sim \text { Amnio otherwise. }
\end{array} .\right.
$$

Note that it is possible that some women will decide to forgo an amniocentesis, even though having a Down syndrome child would reduce their utility, i.e., $\gamma_{1}^{D}<0$. This could occur because while having an amniocentesis allow a woman to avoid having a Down syndrome child with certainty, having the test comes with the risk of losing a healthy child due to a miscarriage.

Before moving to age $T-1$, notice that the choice over amniocentesis only occurs among women pregnant at $T$. Therefore, as of age $T-1$, the payoffs at age $T$ are expected to be 


$$
E\left(u_{T} \mid \gamma_{1}^{D}<\gamma_{T}^{*}\right)=p\left(q+(1-q) \gamma_{1}^{D}\right)
$$

and

$$
E\left(u_{T} \mid \gamma_{1}^{D} \geq \gamma_{T}^{*}\right)=p(q(1-m))
$$

depending on the relationship between a woman's utility from having a Down syndrome child, $\gamma_{1}^{D}$, and its critical value $\gamma_{T}^{*}$ as of age $T$.

Choices at Age T-1-Figure 4 displays the decision tree for a woman at her next-to-last fertile age, $T-1$. At this age, women still have choices they can make when they reach age $T$ and these potential actions have option value as of age $T-1$. Recall that at age $T$ any woman with $\gamma_{1}^{D}<\gamma_{T}^{*}$ would have an amniocentesis and, hence, would abort a pregnancy that tested positive for a chromosomal abnormality. Because the costs of amniocentesis rise with age while the benefits will not so long as $q$ is constant - and the utility associated with not undergoing an amniocentesis is constant in this version of our model, ${ }^{8}$ it follows that $C_{T-1}^{\tau \mid A}=A$ mino and $C_{T-1}^{A \mid \tau=+\& \sim A}=A$ bort when $\gamma_{1}^{D}<\gamma_{T}^{*}$. If $\gamma_{1}^{D} \geq \gamma_{T}^{*}$, the optimal abortion decision rule, at age $T-1$, for a woman who tests positive is given by:

$$
C_{T-1}^{A \mid \tau=+\& \sim A}=\left\{\begin{array}{l}
\text { Abort if } \gamma_{1}^{D}<\left(\frac{p q}{1-p(1-q)}\right) \\
\sim A \text { bort otherwise. }
\end{array}\right.
$$

Since a woman would undergo amniocentesis only if she would abort a fetus that tests positive for a chromosomal abnormality, it follows immediately that

$C_{T-1}^{\tau \sim A}=\sim A m i n o$ if $\gamma_{1}^{D} \geq\left(\frac{p q}{1-p(1-q)}\right)$ and that $\gamma_{T-1}^{*}$ must lie in the interval

$\left[\gamma_{T}^{*},\left(\frac{p q}{1-p(1-q)}\right)\right]$. For all values of $\gamma_{1}^{D}$ in this range, it follows from (11) that a woman would not have amniocentesis at age $T$ should she reach age $T$ without a child and become pregnant at age $T$. Therefore,

$$
E\left(u_{T} \mid C_{T-1}^{\tau \mid \sim A}=A m n i o\right)=m p\left(q+(1-q) \gamma_{1}^{D}\right)+(1-m)\left(q+(1-q) p\left(q+(1-q) \gamma_{1}^{D}\right)\right)
$$

while

$$
E\left(u_{T} \mid C_{T-1}^{\tau \mid \sim A}=\sim A m n i o\right)=q+(1-q) \gamma_{1}^{D}
$$

which implies that

\footnotetext{
${ }^{8}$ Note that with more than one child, the (utility) costs of an amniocentesis will not be constant.
} 


$$
\gamma_{T-1}^{*}=\left(\frac{q}{1-q}\right)\left(\frac{-m+m p+(1-m)(1-q) p}{1-m p-(1-m)(1-q) p}\right)
$$

and the optimal amniocentesis decision-rule at age $T-1$ is:

$$
C_{T-1}^{\tau \mid \sim A}=\left\{\begin{array}{l}
\text { Amnio if } \gamma_{1}^{D}<\gamma_{T-1}^{*} \\
\sim \text { Amnio otherwise. }
\end{array} .\right.
$$

Given that the choice of amniocentesis occurs only among women pregnant at age $T-1$, it follows that the expected future utility as of age $T-2$ is given by one of the following three expressions, depending on a woman's $\gamma_{1}^{D}$ relative to the critical values, $\gamma_{T}^{*}$ and $\gamma_{T-1}^{*}$ :

$$
\begin{aligned}
E\left(u_{T} \mid \gamma_{1}^{D}<\gamma_{T}^{*}\right)+\left(V_{T-1} \mid \gamma_{1}^{D}<\gamma_{T}^{*}\right) & =p\left[m p\left(q+(1-q) \gamma_{1}^{D}\right)+(1-m)\left(q+(1-q) p\left(q+(1-q) \gamma_{1}^{D}\right)\right)\right]+(1-p)[p q(1-m)] \\
E\left(u_{T} \mid \gamma_{T-1}^{*}>\gamma_{1}^{D} \geq \gamma_{T}^{*}\right) & \\
& +\left(V_{T-1} \mid \gamma_{T-1}^{*}>\gamma_{1}^{D} \geq \gamma_{T}^{*}\right) \\
& =p\left[m p\left(q+(1-q) \gamma_{1}^{D}\right)+(1-m)\left(q+(1-q) p\left(q+(1-q) \gamma_{1}^{D}\right)\right)\right] \\
& +(1-p)\left[p\left(q+(1-q) \gamma_{1}^{D}\right)\right]
\end{aligned}
$$

$$
E\left(u_{T} \mid \gamma_{1}^{D} \geq \gamma_{T-1}^{*}\right)+\left(V_{T-1} \mid \gamma_{1}^{D} \geq \gamma_{T-1}^{*}\right)=p\left[\left(q+(1-q) \gamma_{1}^{D}\right)+(1-p)\left(q+(1-q) \gamma_{1}^{D}\right)\right] .=(2-p)\left[p\left(q+(1-q) \gamma_{1}^{D}\right)\right]
$$

Choices at Age $\boldsymbol{T}-2$ and Arbitrary Ages $\boldsymbol{T}-\boldsymbol{s}-$ Continuing with this recursive solution strategy at age $T-2$, recall that at age $T-1$ any woman with $\gamma_{1}^{D}<\gamma_{T-1}^{*}$ would have an amniocentesis and hence abort a pregnancy that tested positive for a chromosomal abnormality. Again, because the costs of amniocentesis rise with age while the benefits do not (when $q$ is constant), this immediately implies that $C_{T-2}^{\tau \mid \sim A}=A m n i o$ and $C_{T-2}^{A \mid \tau=+\& \sim A}=A b o r t$ for $\gamma_{1}^{D}<\gamma_{T-1}^{*}$ or, for an arbitrary age, $T-S$, $C_{T-s}^{\tau \mid A}=A m n i o$ and $C_{T-s}^{A \mid \tau=+\& \sim A}=A$ bort for $\gamma_{1}^{D}<\gamma_{T-s+1}^{*}$. At age $T-2$, for $\gamma_{1}^{D} \geq \gamma_{T-2}^{*}$, the abortion decision rule for women who test positive is given by:

$$
C_{T-2}^{A \mid \tau=+\& \sim A}=\left\{\begin{array}{l}
A b o r t \text { if } \gamma_{1}^{D}<\left(\frac{p(1+(1-p)) q}{1-p(1+(1-p))(1-q)}\right) \\
\sim A b o r t \text { otherwise. }
\end{array}\right.
$$

or, at an arbitrary age, $T-s$, by: 


$$
C_{T-s}^{A \mid \tau=+\& \sim A}=\left\{\begin{array}{l}
\text { Abort, if } \gamma_{1}^{D}<\left(\frac{p\left(\sum_{j=0}^{s-1}(1-p)^{j}\right) q}{1-p\left(\sum_{j=0}^{s-1}(1-p)^{j}\right)(1-q)}\right)=\left(\frac{\left(1-(1-p)^{s}\right) q}{\left(1-\left(1-(1-p)^{s}\right)(1-q)\right)}\right) . \\
\sim \text { Abort, otherwise. }
\end{array}\right.
$$

Since a woman would undergo amniocentesis only when she would abort a fetus that tests positive for a chromosomal abnormality, this immediately implies that

$$
C_{T-s}^{\tau \mid \sim A}=\sim A \text { mnio if } \gamma_{1}^{D} \geq\left(\frac{\left(1-(1-p)^{S}\right) q}{\left(1-\left(1-(1-p)^{s}\right)(1-q)\right)}\right)
$$

And, thus, $\gamma_{T-s}^{*}$ must lie in the interval, $\left[\gamma_{T-s+1}^{*},\left(\frac{\left(1-(1-p)^{s}\right) q}{\left(1-\left(1-(1-p)^{s}\right)(1-q)\right)}\right)\right]$. For all $\gamma_{1}^{D}$ in this range, a woman would not have amniocentesis at any (fertile) age after $T-s$. Therefore,

$$
E\left(u_{T-s} \mid C_{T-s}^{\tau \mid \sim A}=A m n i o\right)=(1-m) q+(m+(1-m)(1-q))\left(p\left(q+(1-q) \gamma_{1}^{D}\right)\right)\left(\sum_{j=0}^{s}(1-p)^{j}\right),
$$

while

$$
E\left(u_{T-s} \mid C_{T-s}^{\tau+\sim A}=\sim A m n i o\right)=q+(1-q) \gamma_{1}^{D}
$$

Thus, the critical value for $\gamma_{1}^{D}$ for an arbitrary age $T-s$ is given by

$$
\gamma_{T-s}^{*}=\left(\frac{q}{1-q}\right)\left(\frac{-m+\theta\left(1-(1-p)^{s}\right)}{1-\theta\left(1-(1-p)^{s}\right)}\right) \text {, }
$$

where $\theta \equiv(m+(1-m)(1-q)) \geq 0$, and optimal decision-rule for a woman's amniocentesis choice at that age is

$$
C_{T-s}^{\tau \mid \sim A}=\left\{\begin{array}{l}
\text { Amnio if } \gamma_{1}^{D}<\gamma_{T-s}^{*} \\
\sim \text { Amnio otherwise }
\end{array}\right.
$$

Predictions for the Simplified Model-What are the dynamic patterns of choices, especially with respect to amniocentesis, predicted by this simple model? Given the form of the decision rule for a woman's amniocentesis choice at any age $T-s$ in (21), how amniocentesis choice varies with maternal age depends upon how $\gamma_{T-s}^{*}$ varies with age, given the single-crossing property of (21). Therefore, when $s=0$ (at age $T$ ), $\gamma_{T}^{*}=\left(\frac{q}{1-q}\right)(-m$ ) and as $t \rightarrow T$ (as a woman approaches menarche), $\gamma_{T-s}^{*} \rightarrow 1$. That is, our simplified model implies that all young women would choose to undergo an amniocentesis and that the incidence of amniocenteses should decline monotonically with age. 
Clearly, the above prediction with respect to how amniocentesis varies with maternal age is inconsistent with the advice given by the medical community. But how does it compare with the pattern for observed life cycle patterns of amniocenteses? To address this issue, and to determine what features of our more general model of amniocentesis choice need to be modified to "fit the data," we present, in the following section, evidence on the empirical relationship between amniocentesis and age.

\section{Empirical Regularities on Amniocentesis and Maternal Age}

To investigate the relationship between age and amniocentesis, we use data from the Detailed Natality Files (DNF) from the National Center for Health Statistics. The DNF include all births occurring within the United States. Beginning in 1988, for nearly every state, both congenital anomalies and a woman's use of amniocenteses during pregnancy are recorded. ${ }^{9}$ While some twenty-two congenital anomalies are recorded, many are not genetic in origin. ${ }^{10}$ In what follows, we concentrate on the data for three anomalies that are routinely discovered by amniocentesis: spina bifida, Down's syndrome, and "other chromosomal anomalies." These anomalies comprise more than half of all genetic disorders associated with newborns.

The benefit of the DNF is that it is a census of births in the U.S. and provides sufficient sample size to study choices of women pregnant at older ages. One potential issue is that this is a sample of live births only and hence Down's syndrome fetuses that are aborted do not appear in the sample. However, it is possible to estimate the fraction of pregnancies for which amniocentesis is performed from the sample of live births.

From the DNF, we can obtain the following statistics:

$\begin{array}{ll}N_{r t}^{\text {Birth }}= & \text { Total number of births of parity } r \text { occurring to women of age } t ; \\ N_{r t}^{B \text { Birth\&Ammio }}= & \text { Total number of births of parity } r \text { preceded by an amniocentesis occurring to women of age } t ;\end{array}$

and

$$
N_{r t}^{\text {Birth\& } \sim A m n i o}=N_{r t}^{\text {Birth }}-N_{r t}^{\text {Birth\&Amnio } .}
$$

The rate of amniocenteses conditional on live births is defined to be:

$$
\operatorname{Pr}\left(\text { Amnio }_{r t} \mid b_{r t}=1\right)=\frac{N_{r t}^{\text {Birth\&Amnio }}}{N_{r t}^{\text {Birth }}},
$$

where $b_{r t}=1$ indicates a live birth of birth order $r$ to a woman of age $t$.

For our purposes, we are interested in the rate of amniocentesis conditional on pregnancies and only on "relevant" pregnancies. In particular, an amniocentesis is not a relevant choice for a woman who would elect to abort a pregnancy, without regard to its genetic status, or for a woman who has a naturally occurring miscarriage prior to the amniocentesis choice. ${ }^{11}$

\footnotetext{
${ }^{9}$ The following states did not record amniocentesis on a significant percentage of State Birth Certificates (between 1990 and 1994): Connecticut (16.6\%), Maryland (20.4\%) and Oklahoma (35.0\%). The following states did not record at least one of the three abnormal conditions of the newborn on a significant percentage of State Birth Certificates: Rhode Island (20.08\%), Connecticut (26.5\%), Maryland (33.21\%), Oklahoma (42.49\%), New York (92.07\%), and New Mexico (100\%).

${ }^{10}$ These include central nervous, cardiac, musculoskeletal, and gastrointestinal.
} 
We term relevant pregnancies as those that are wanted and viable and define them to include: (a) pregnancies ending in births that are not preceded by an amniocentesis $\left(N_{r t}^{\text {Birth\& } \sim A m n i o}\right.$ ); (b) pregnancies resulting in births that are preceded by an amniocentesis $\left(N_{r t}^{\text {Birth\&Amnio }}\right.$; (c) pregnancies followed by an amniocentesis that was positive and was

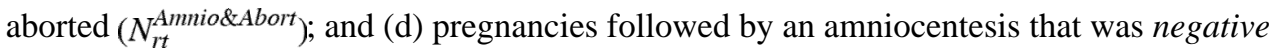
but which induced a miscarriage $\left(N_{r t}^{\text {Ammio\&Miscarry }}\right)$. It follows that the total number of wanted and viable pregnancies is equal to

$$
\begin{aligned}
N_{r t}^{W} & =N_{r t}^{\text {Birth\& } \sim \text { Amnio }}+N_{r t}^{\text {Birth\&Amnio }}+N_{r t}^{\text {Amnio\&Abort }}+N_{r t}^{\text {Amnio\&Miscarry }} \\
& =N_{r t}^{\text {Birth\& } \sim \text { Amnio }}+N_{r t}^{\text {Amnio }} q_{t}(1-m)+N_{r t}^{\text {Ammio }}\left(1-q_{t}\right)+N_{r t}^{\text {Ammio }} q_{t} m, \\
& =N_{r t}^{\text {Birth\& } \sim \text { Amnio }}+N_{r t}^{\text {Amnio }}
\end{aligned}
$$

where $\left(1-q_{t}\right)$ is the rate of chromosomal abnormalities at age $t, m$ is the rate of miscarriage induced by the amniocentesis procedures, and $N_{r t}^{A m m i o}$ is the number of amniocentesis procedures performed. The substitutions of

$N_{r t}^{\text {Ammio }} q_{t}(1-m)$ for $N_{r t}^{\text {Birth\&Ammio }}$ and $N_{r t}^{\text {Ammio }}\left(1-q_{t}\right)$ for $N_{r t}^{\text {Abort\&Amnio }}$ in the second line of (23) follow from invoking the following rational choice assumption: women who test positive for a chromosomal abnormality will always choose to abort their fetuses, while women who test negative will always choose to bring their fetuses to term. The DNF suggests that it is very rare that a woman give birth to a Down syndrome child after receiving amniocentesis. ${ }^{12}$ Finally, we substitute $N_{r t}^{\text {Ammio }} q_{t} m$ for $N_{r t}^{\text {Amnio\&Miscarry }}$ in (23) to reflect the fact that technical problems can cause an amniocentesis to end in a miscarriage at the rate $m$.

It follows that the rate of amniocenteses conditional on wanted and viable pregnancies is given by

$$
\operatorname{Pr}\left(\text { Amnio }_{r t} \mid p_{r t}^{W}=1\right)=\frac{N_{r t}^{A m n i o}}{N_{r t}^{W}}
$$

where Amnio $r t$ is the indicator on whether a woman had an amniocentesis at age $t$ for parity $r$ and $p_{r t}^{W}$ is an indicator that a pregnancy was wanted and viable. Recall that

$N_{r t}^{\text {Birth\&Amnio }}=N_{r t}^{\text {Amnio }} q_{t}(1-m)$, or that $N_{r t}^{\text {Amnio }}$ can be estimated as $N_{r t}^{\text {Amnio }}=\frac{N_{r t}^{\text {Birth\&Amnio }}}{q_{r}(1-m)}$. Furthermore, recall that $N_{r t}^{W}=N_{r t}^{\text {Birth\& } \sim A m n i o}+N_{r t}^{A m n i o}$. It follows that that equation (24) can be expressed as

$$
\operatorname{Pr}\left(\text { Amnio }_{r t} \mid p_{r t}^{W}=1\right)=\frac{\frac{N_{r t}^{\text {Birh } \& \text { Amnio }}}{q_{t}(1-m)}}{N_{r t}^{\text {Birth\& } \& \text { Amnio }}+\frac{N_{r t}^{\text {Birh \&Amnio }}}{q_{t}(1-m)}}
$$

\footnotetext{
${ }^{11}$ There are a variety of estimates for the rate of fetal loss by gestational age. Taylor (1969) estimates the rate of loss (per 1000 pregnancies) by gestational age is approximately 28 ( $<7$ weeks), 45 (8-11 weeks), 20 (12-15 weeks) and less than 10 after the $15^{\text {th }}$ week. Taylor's estimates imply that $72 \%$ of miscarriages occur prior to week 16 of pregnancy. Amniocentesis is not performed prior to week 16.

12 Among the live births that were reported to have Down syndrome between 1990 and 1994, 9.5\% were preceded by an amniocentesis, while $90.5 \%$ were not.
} 
where $N_{r t}^{\text {Birth\&Ammio }}$ and $N_{r t}^{\text {Birth\& } \sim \text { Ammio }}$ are obtained from the DNF and we derive values for $q_{t}$ and $m$ from medical studies.

Clearly, the rates in (25) may vary by more than just a woman's parity $(r)$ and age $(t)$. For example, these rates may vary by her education or race. For example, because Down's syndrome children are time intensive, one might expect that more educated women, who tend to have higher market-based opportunity costs of time, have higher rates of amniocentesis than less educated women. Further, more educated women may differ in the likelihood of becoming pregnant by birth parity and age, i.e., women having a first birth late in life are likely to have high levels of education. To deal such factors, one can calculate separate rates by these demographic characteristics. Alternatively, one can "hold constant" other covariates when calculating (25) by weighting one group to mimic the distribution of covariates of another. For example, we might standardize all of our age-specific estimates to the educational distribution for women who have a first birth at say age 21 in order to hold education constant across age. Then our standardized (by education) age-specific estimate of the rate of amniocenteses would be

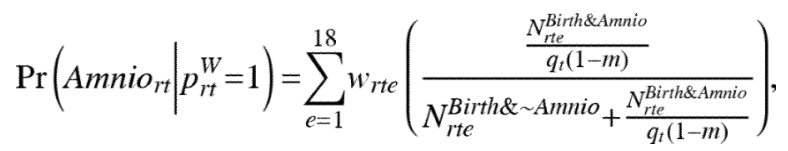

where $w_{r t e}=\frac{k_{1,21, e}}{k_{r t e}}, k_{r t e}=\frac{N_{r t e}}{N_{r t}}$, and $e$ indexes education groups.

Figure 5 plots $\operatorname{Pr}\left(\right.$ Amnio $\left.o_{r t} \mid p_{r t}^{W}=1\right)$ for maternal ages 20 to 48 and for first through fourth parities. Several interesting patterns emerge from this Figure. First, regardless of parity, the rate of amniocentesis is near zero for women under the age of 35, but there is a steep rise in the rate of amniocentesis after that age for all parities. Second, the increase in rate of amniocentesis is larger the lower the parity of the child. Finally, while amniocentesis rates rise until a woman reach her mid-forties, they begin to decline after that age and they drop more precipitously for lower parities (first through third) than higher (fourth) parities. In particular, between the ages of 46 to 48 , the rate of amniocentesis drops from $31 \%$ to $20 \%$ for first births, from $28 \%$ to $14 \%$ for second births, and from $45 \%$ to $23 \%$ for third births. In contrast, there is very little decline in the corresponding rates for fourth births over this same age range.

These age and parity patterns in rates of amniocentesis may be the result of changes in the composition of women across these groups. As noted above, women having a first birth at age 20 are likely to be less educated than those having their first birth at age 40 . One easy way to account for this compositional effect on these rates is to condition, non-

parametrically, on the educational attainment of women. Figure 6 plots $\left.\operatorname{Pr}^{(A m n i o r t} \mid p_{r t}^{W}=1\right)$ for maternal ages 20 to 48 and for first through fourth parities for women whose highest level of educational attainment was a high school diploma. What is clear is that the patterns described for all women persist although the differences in the rates at any age across parity are reduced. Figure 7 plots $\left.\operatorname{Pr}^{\left(A m n i o_{r t}\right.} \mid p_{r t}^{W}=1\right)$ when the distribution of race, marriage and education is held constant for all parities and for all ages at the distribution found for women having a second birth at age $35 .{ }^{13}$ Again, for the first through third children, the patterns remain. 
In summary, the data on the rate of amniocentesis in Figures 5 through 7 display the following patterns:

- Almost no women undergo an amniocentesis prior to age 35 .

- The rate at which women undergo the procedure rises rapidly after age 35 , rising more the lower the child parity.

- At every parity, the rate of amniocentesis declines as a woman approaches the end of her reproductive years.

Presumably, the first and second of these patterns are heavily influenced by the "rules" that health care providers and insurance companies follow with respect to what ages they advise women to have an amniocentesis and the age-related restrictions that health care providers and insurers place on the reimbursements of this procedure that were noted in the Introduction. But it is less clear how to account for the third pattern, namely, that the incidence of amniocenteses by birth parity decline towards the end of woman's reproductive lives.

As we already have seen, a simple version of a life cycle model of optimal amniocentesis choice appears to be inconsistent with the first two of the above life cycle patterns observed in the data for amniocenteses, although it does tend to characterize the third one. In the following section, we consider the life cycle patterns that are generated by more complicated versions of our dynamic model, examining how the model's predictions concerning life cycle variation in the likelihood of having an amniocentesis change as we vary the rate of discounting, the utility costs of abortions and miscarriages and with age-variation in the rates of genetic disorders, such as displayed in Figure 1. We contrast these predictions with those generated by a myopic model of amniocentesis choice, exploiting the fact that such a model arises as a special case of our dynamic model under the case where the future is completely discounted (i.e., $\beta=0.0$ ) when women (couples) make their childbearing and amniocentesis decisions.

\section{Varying Features of the Model and Tracking the Observed Dynamic Patterns of Amniocentesis Choice}

In this section, we examine how changes in the various features of our dynamic model of amniocentesis choice affect our ability to "replicate" the age patterns found in the data on the incidence of amniocenteses described in the preceding section. We pay particular attention to contrasting our dynamic model with the special case in which women are myopic with respect to these choices, i.e., do not take full account of the future in making their choices. In this exploration, our goal is not to fit our model to data in a statistical sense. Rather, our goal is to see whether our model, and its underlying features, is rich enough to characterize the dynamic patterns of amniocentesis choice outlined above. We proceed by plugging in values for the key parameters (or rates) for the model in Section 2 in an attempt to "calibrate" the model, assessing how well we mimic the dynamic patterns of amniocentesis choice at different ages by plotting how the critical value, $\gamma_{t}^{*}$, for $\gamma_{1}^{D}$ varies with age. ${ }^{14}$ To simplify our analysis, we normalize the value of a (chromosomally) healthy

\footnotetext{
${ }^{13}$ Blacks, Hispanic, Others and Whites make up $11.0 \%, 11.6 \%, 6.0 \%$ and $72.4 \%$ of this population respectively. High School dropouts, high school graduates, some college and college graduates make up $14.4 \%, 26.6 \%, 22.7 \%$, and $40.3 \%$ respectively of this population respectively. $85.6 \%$ of this population is married. Figure 7 is constructed through regression adjustments and then equation (25) is applied. In future versions of this paper these will be constructed entirely non-parametrically.

${ }^{14}$ That is, the incidence of amniocenteses at a woman's age, $t$, is given by $\operatorname{Pr}\left(\gamma_{1}^{D} \leq \gamma_{t}^{*}\right)$ in decision-rule (21), where $\gamma_{1}^{D}$ is treated as a random variable with a known distribution.
} 
child to one $\left(\gamma_{1}^{N}=1\right)$, and specify the following parameters of the model to be age-invariant and equal to the following values: annual pregnancy rate $(p)$ to 0.3 and the rate of procedureinduced miscarriage $(m)$ to $0.0067(1 / 150)$. We then explore how varying the following parameters affects $\gamma_{t}^{*}$ and thus the incidence of amniocenteses with age: (i) the discount rate ( $\beta$ ); (ii) the utility cost of an abortion $(\alpha)$; (iii) the utility cost of a miscarriage ( $\mu$ ); and (iv) the rate of genetic disorders (Down syndrome) $(q)$, where for the latter parameter we consider specifications in which $q$ is assumed to be age-invariant and to vary with age as in Figure 1.15

The results of this exploration of our model are displayed in Figures 8 through 12. Consider first the role that discounting plays in affecting the dynamic pattern of $\gamma^{*}$, and, thus, the incidence of amniocenteses. Figure 8 displays how $\gamma^{*}$ varies with age assuming that the rate of genetic disorders associated with births is age-invariant (i.e., $q_{a}=q$ for all $a$ ) and there are zero (utility) costs of abortions and miscarriages (i.e., $\alpha=\mu=0$ ). Consider first the values for $\gamma^{*}$ when $\beta=0.0$, i.e., the "myopic" model. In our characterization of the myopic model, a woman is assumed to not take account of the possibility of "replacing" a current pregnancy with a future pregnancy in her decision to get amniocentesis because she completely ignores the future. However, we do assume that a woman takes account of the "durable" nature of children and of abortion and miscarriage costs in making her childbearing, amniocentesis and abortion decisions, via the "payoff values" $\gamma^{D}, \alpha$ and $\mu$. That is, we assume that women still take account of the PDU she receives from her child and the PDU associated with any abortion or miscarriage she experiences.

As can be seen in Figure 8, the value for $\gamma^{*}$ at early ages increases-and thus the probability of having an amniocentesis increases, as we move from the myopic version of the model to the two variants of the Dynamic Model $(\beta=0.50$ and $\beta=0.95)$. This increase reflects the "option value" of knowing about the genetic status of a fetus at early ages. When, in the Dynamic Model, the future is taken into account in decision-making, forward looking women have an incentive to learn about the genetic status of a fetus precisely because they realize that they have the option of aborting such a fetus if the amniocentesis is positive and replacing that fetus in the future. Women under the Myopic Model do not consider this option and, thus, have no greater incentive to have an amniocentesis at early ages than they do at older ages. Note, too, that the less a woman discounts the future-i.e., comparing the results for $\beta=0.95$ with those for $\beta=0.50$-the greater is this incentive to have an amniocentesis at early ages. Finally, as women age, there is no change in the value of $\gamma^{*}$ under the Myopic model, but this critical value declines with age under either specification of the Dynamic Model. Furthermore, $\gamma^{*}$ converges to the same value under all three specifications at age $T$, the end of the woman's reproductive life. This convergence follows from the fact that the decision rule at age $T$ is identical under both the Dynamic and Myopic model, since there is no future at that age.

In addition, this decline in $\gamma^{*}$ with age under the Dynamic specification reflects another force within our model. As noted above, the higher the $\gamma_{1}^{D}$ (and $\gamma_{1}^{N}$ ), the greater the desire of women to have a child, even if it is one that has Down syndrome. As women approach the end of their reproductive careers, their incentive to have a child increases under the Dynamic specification. As a result, childless women have an incentive to avoid actions that further

\footnotetext{
${ }^{15} \mathrm{An}$ annual rate of pregnancy of 0.3 is consistent with data from the Hutterites. The average number of births to fecund women in this population is approximately 11 . In our simulations, we use this age-invariant rate, despite clear evidence that pregnancy rates tend to fall over time (see, for example, Leridon, 2008). Allowing for a monotonically declining pregnancy rate with maternal age would tend to exacerbate the life-cycle effect, i.e. as women approach menopause without having reached the desired family size, the disutility from an amniocentesis-induced miscarriage would be greater as the probability of becoming pregnant again would be declining over time.
} 
postpone births. This includes undergoing an amniocentesis, since the latter procedure is assumed to increase the likelihood that a woman experiences a miscarriage. Thus, as one approaches $T$, forward-looking women will have less incentive to undergo an amniocentesis in order to reduce the risk of a miscarriage, which would further postpone their chances of having a birth prior to menopause. Note, that this age-dependent incentive is not present in the Myopic model, precisely because it does not have women (couples) be forward looking when making their choices over the life cycle.

In Figure 9, the only change in the specifications of either the Dynamic or Myopic models is to allow the probability of a genetic disorder to vary with age, following the pattern in Figure 1. Several interesting implications emerge by comparing the age patterns for $\gamma^{*}$ displayed in this Figure 8 and Figure 9. First, both figures make clear that forward-looking women have a greater incentive to have an amniocentesis at early ages compared to optimal choices under the Myopic Model. Second, when women face a rising (with age) risk of having a child having a genetic disorder, under either the Dynamic or Myopic model, their incentive to have an amniocentesis is lowered at early ages. In the Myopic model, the rising pattern to $\gamma^{*}$ simply reflects a age-by-age response to the increasing risk of a birth defect with age; as the risk of a birth defect goes up, the incentives to learn about the potential problems with a fetus rise. With respect to the Dynamic models, the consequences of rising rates of birth defects for the age pattern in $\gamma^{*}$ depend on the extent of discounting. At the extreme, if women do not discount the future at all, i.e., $\beta=1.00$, the likelihood of an amniocentesis does not rise with age. Rather, the option value effect noted above dominates her (their) amniocentesis decisions, giving rise to high rates of amniocentesis at early ages, while the risks of miscarriage associated with an amniocentesis, causes their utilization to decline as the woman (couple) reaches the end of the childbearing period.

In Figures 10 and 11, we examine how the predictions of our Myopic and Dynamic models, respectively, are affected by changes in the utility costs of abortions and costs. As can be seen from these figures, higher costs to either abortion reduces the critical value for amniocenteses equally at all ages while higher costs for miscarriages tend to reduce the incidence of amniocenteses more at younger ages than at older ones.

Finally, in Figure 12, we summarize our findings by graphing the life cycle pattern of $\gamma^{*}$ for both our Dynamic and Myopic models, for both age-invariant and age-varying rates of genetic disorders $(1-q)$. While more work needs to be done in "calibrating" the Dynamic models, ${ }^{16}$ this figure suggests that a Dynamic model in which there is some, but not complete, discounting of the future and which incorporates the age-varying rates of birth defects $(1-q)$ displayed in Figure 1 can generate the qualitative features of the observed agepatterns in the incidence of amniocenteses. In particular, such a model is able to generate both the rise in the incidence of amniocentesis with age and the decline in its utilization at older ages. In contrast, the Myopic model we calibrate does not generate a decline in $\gamma_{T-s}^{*}-$ and, thus, in the incidence of amniocenteses - as women approach menopause.

\section{Concluding Observations}

In this article, we examine the appropriateness of the threshold-age approach in the protocol for recommending amniocentesis testing to pregnant women, based on a dynamic model of the decision making process. In particular, we consider the potentially welfare enhancing effect of fully informing women of the risks and benefits of such a procedure, irrespective of age.

\footnotetext{
${ }^{16}$ In particular, it would appear that we can achieve age-patterns that more closely accord with those in the actual data for amniocenteses displayed in Figures 5 through 7 by increasing some combination of the disutility costs of miscarriages or abortions.
} 
Based on our calibration exercise, it does appear that the features of our model may be important in accounting for the observed life cycle pattern of amniocenteses. In particular, the dynamic model of optimal amniocentesis choice outlined above, when it includes some, but not total, discounting of the future and conditions on increasing rates of genetic disorders with age, does appear to generate age-patterns consistent with the data. Moreover, it appears that Myopic models fail to predict the decline in the incidence of amniocentesis with age that are found in the data. While further exploration of the calibration of the Dynamic model is needed (for instance, to account for endogenous desired family size or an age-dependent pregnancy rate) these initial findings suggest real merit to develop and estimate a dynamic structural model of amniocentesis choice of the sort outlined in this article.

These initial findings, if they hold up to further scrutiny, have potentially important implications for medical practice and insurance company policies for the funding of this procedure. In particular, our results suggest that the medical community and insurance companies may be ignoring the option value of amniocenteses when women are young. Currently, $80 \%$ of Down syndrome children are born to women under the age of 35 . Our model suggests that some of these young women, if informed of the full costs and benefits of the procedure and given the option to have it, would undergo an amniocentesis and, upon learning of the results of this test, would choose to abort a chromosomally abnormal fetus in favor of a future pregnancy that could result in a healthy birth. As such, existing guidelines of health care providers and insurance companies in setting policies for the ages at which amniocenteses will be funded are potentially inconsistent with the choices some women would make for themselves if free to acquire the information provided by this procedure. Further exploration and estimation of this model may help to inform medical practice and policy by explicitly accounting for choice behavior among women (couples) that take account of the future consequences of their actions.

\section{Acknowledgments}

This research was funded by a grant from the National Institute for Child Health and Human Development (R01HD34293). Obviously, only the authors are responsible for the content of this paper. We would like to thank Margo Prator for suggesting this topic.

\section{References}

Ben-Porath Y. Fertility response to child mortality: Micro data from Israel. Journal of Political Economy. 1976; 84:S163-S178.

Dick PT. with the Canadian Task Force on the Periodic Health Examination. Periodic health examination, 1996 update: 1. Prenatal screening and diagnosis of Down syndrome. Canadian Medical Association Journal. 1996; 154:465-479. [PubMed: 8630836]

Hook EB. Rates of chromosomal abnormalities at different maternal ages. Obstetrics and Gynecology. 1991; 58:282-285. [PubMed: 6455611]

Hook EB, Cross PK, Schreinemachers DM. Chromosomal abnormality rates at amniocentesis and in live-born infants. Journal of the American Medical Association. 1983; 249:2034-2038. [PubMed: 6220164]

Leridon $\mathrm{H}$. A new estimate of permanent sterility by age: sterility defined as the inability to conceive. Population Studies. 2008; 62:15-24. [PubMed: 18278670]

National Center for Health Statistics. Monthly Vital Statistics Report. Vol. 42. Hyattsville, MD: Public Health Service; 1994. Advance report of maternal and infant health from the birth certificate, 1991.

Preston, SH. Introduction. In: Preston, SH., editor. The effects of infant and child mortality on fertility. New York: Academic Press; 1978. p. 1-18.

Sah RK. The effects of child mortality and fertility choice and parental welfare. Journal of Political Economy. 1991; 99:582-606. 
Shultz TP. An economic model of family planning and fertility. Journal of Political Economy. 1969; 77:153-180. [PubMed: 11619428]

Shultz, TP. Interrelationships between mortality and fertility. In: Ridker, RG., editor. Population and development: The search for Selective Interventions. Baltimore, MD: John Hopkins University Press; 1976. p. 239-289.

Sicherman N, Bombard AT, Rappoport P. Current maternal age recommendations for prenatal diagnosis: Reappraisal using the expected utility theory. Fetal Diagnosis and Therapy. 1995; 10:157-166. [PubMed: 7639936]

Taylor, WF. The probability of fetal death. In: Fraser, FC.; McKusic, VA., editors. Congenital Malformations. New York: Excerpta Medica; 1969. p. 307-332.

U.S. Preventative Service Task Force. Guide to Clinical Preventive Services. 2nd ed.. Baltimore, MD: Williams \& Wilkins; 1996. Screening for Down syndrome.

Waitzman NJ, Romano PS, Scheffler RM. Estimates of the economic costs of birth defects. Inquiry. 1994; 31:188-205. [PubMed: 8021024]

Wolpin, K. The determinants of infant and child mortality and their consequences for fertility. In: Rosenzweig, M.; Stark, O., editors. Handbook of Population and Family Economics. Amsterdam, Netherlands: North-Holland; 1997. p. 483-557. 


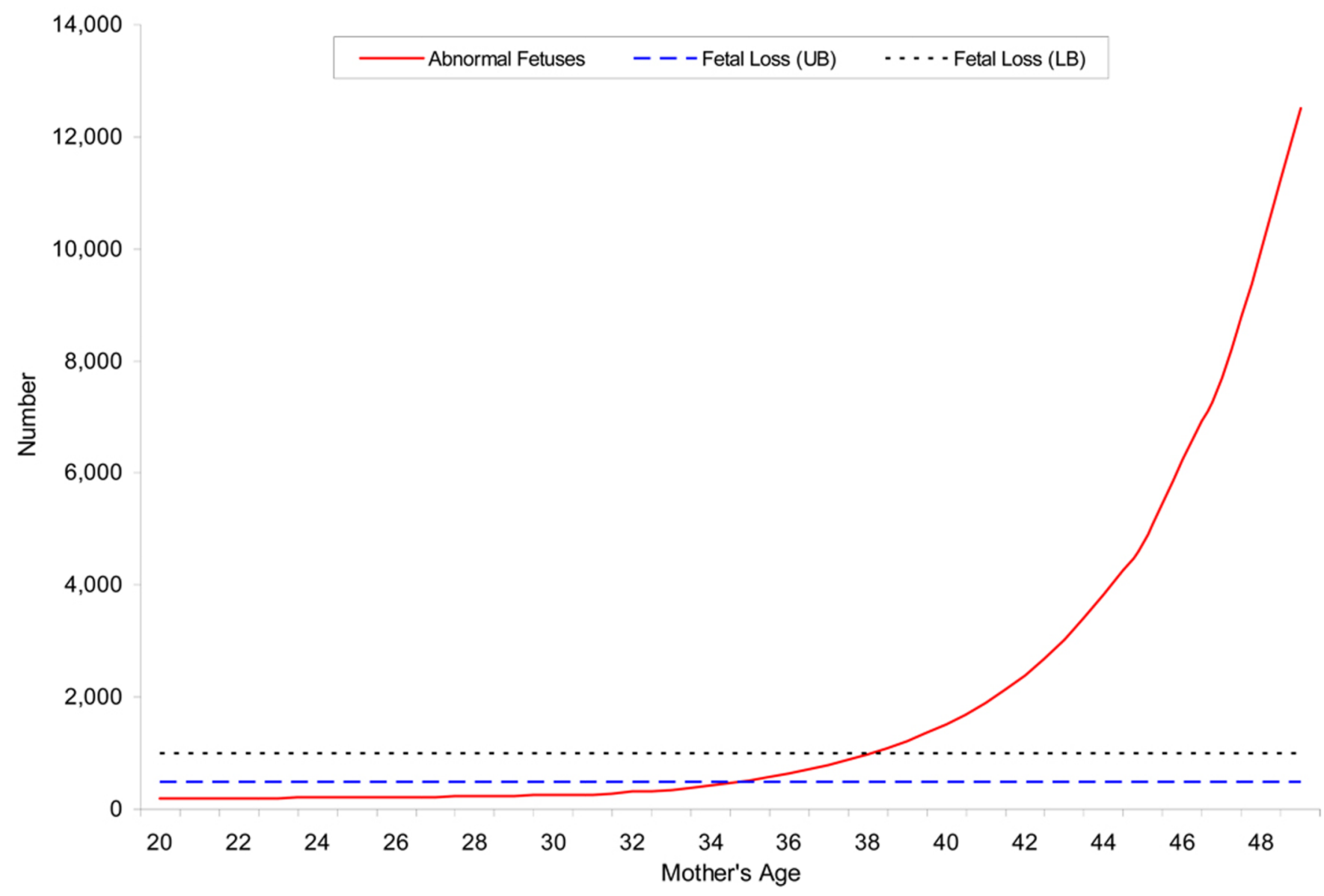

Fig. 1.

Number of Chromosomally Abnormal Fetuses per 100,000 Pregnancies [Sources: Hook (1981); and Hook, Cross \& Schreinemachers (1983) and U.S. Preventative Service Task Force (1996)] 


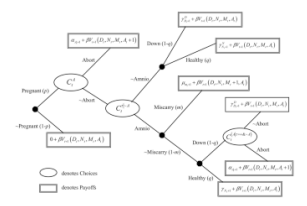

Fig. 2.

Decision tree for general model at (mother's) age $t$ 


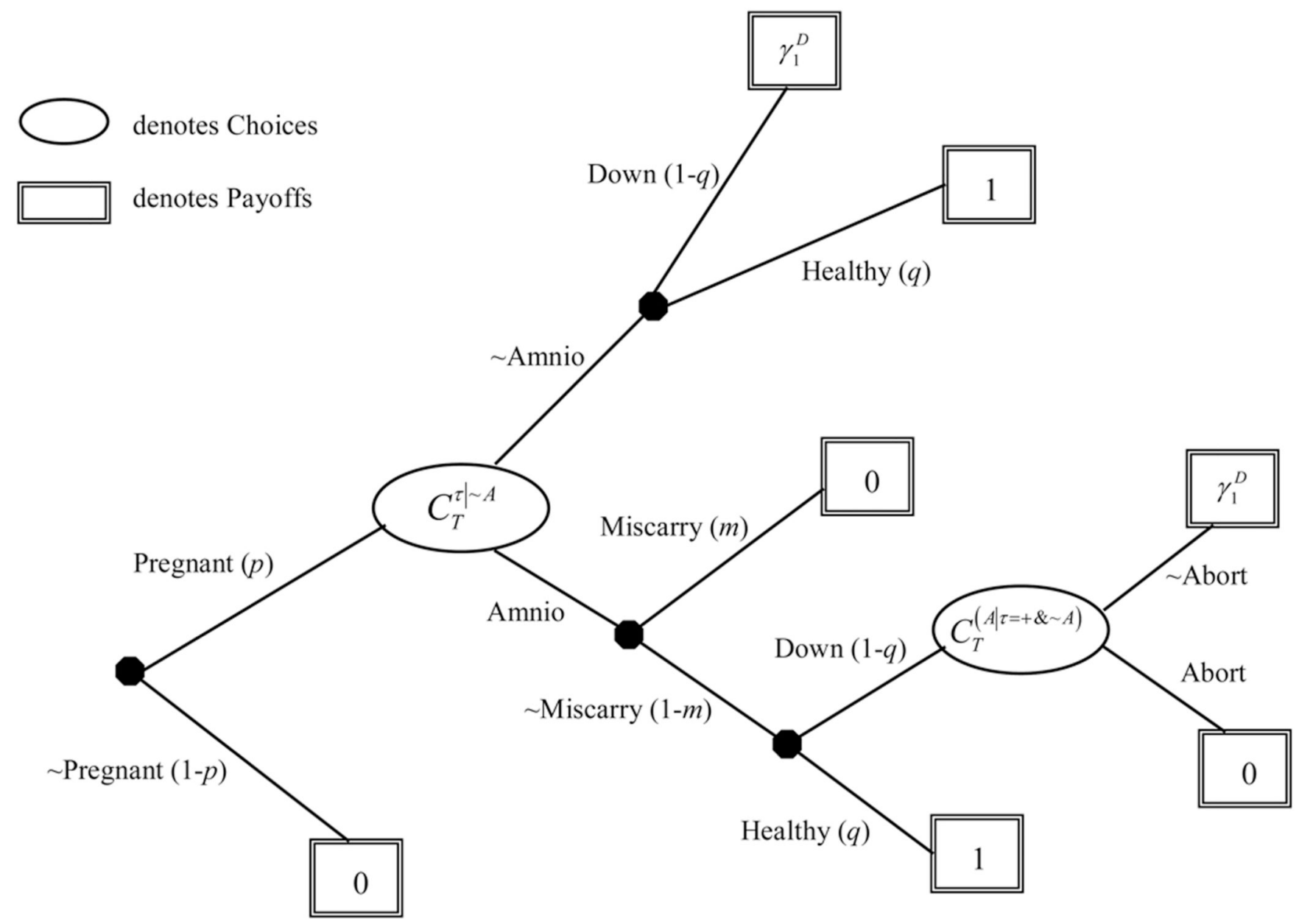

Fig. 3.

Decision tree for simplified model at age $T$ 


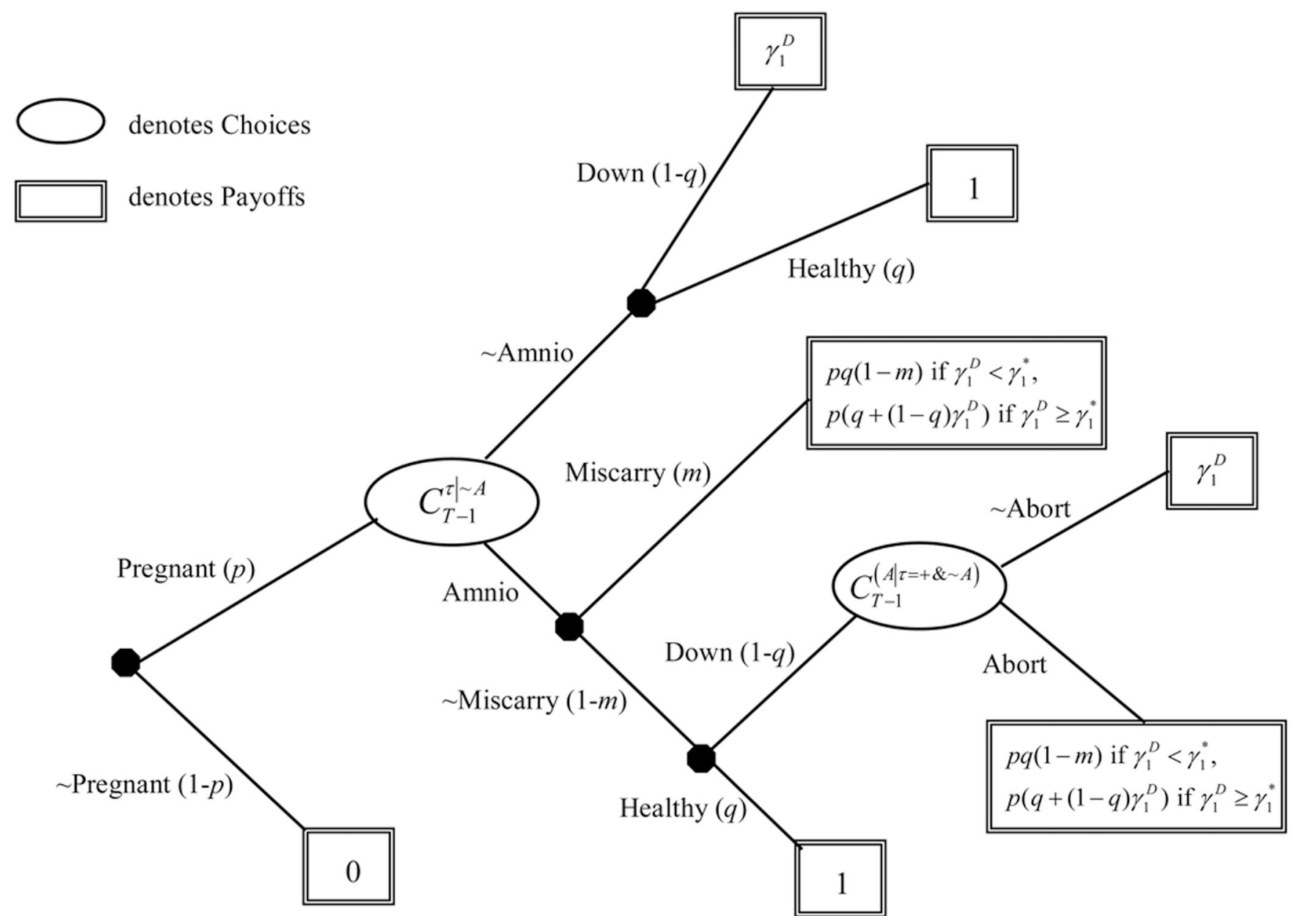

Fig. 4.

Decision tree for simplified model at age $T-1$ 


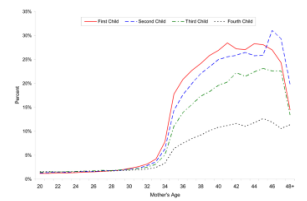

Fig. 5.

Percent of estimated wanted and viable pregnancies that undergo amniocentesis, All mothers [Data: NCES Detailed Natality Files, 1990-1994 Pooled] 


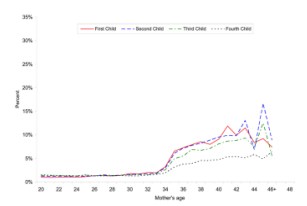

Fig. 6.

Percent of wanted and viable pregnancies that undergo amniocentesis, Mothers with yrs. of school $\leq 12$ [Data: NCES Detailed Natality Files, 1990-94 Pooled] 


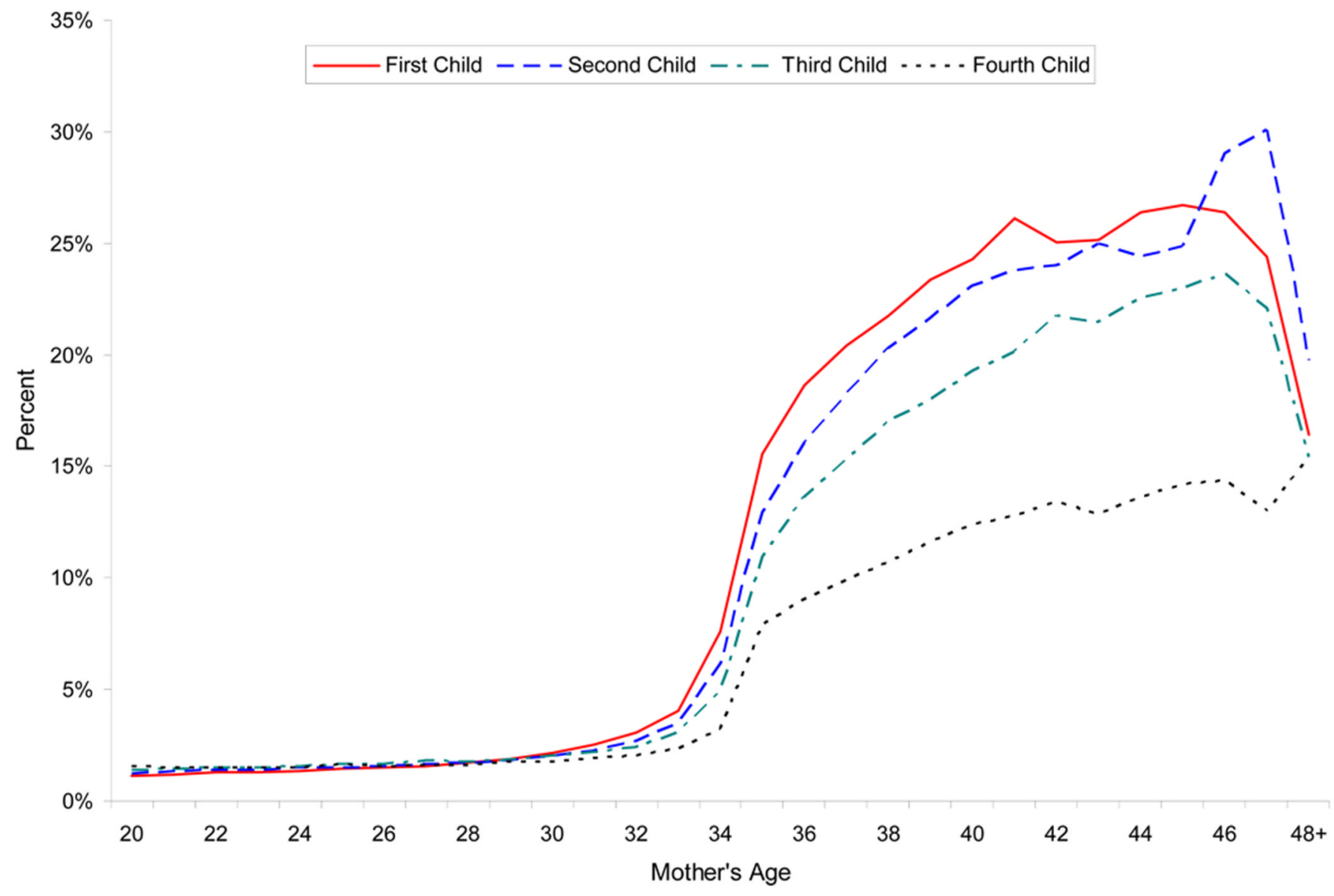

Fig. 7.

Percent of wanted and viable pregnancies that undergo amniocentesis, Regression Adjusted [NCES Detailed Natality Files, 1990-94 Pooled] 


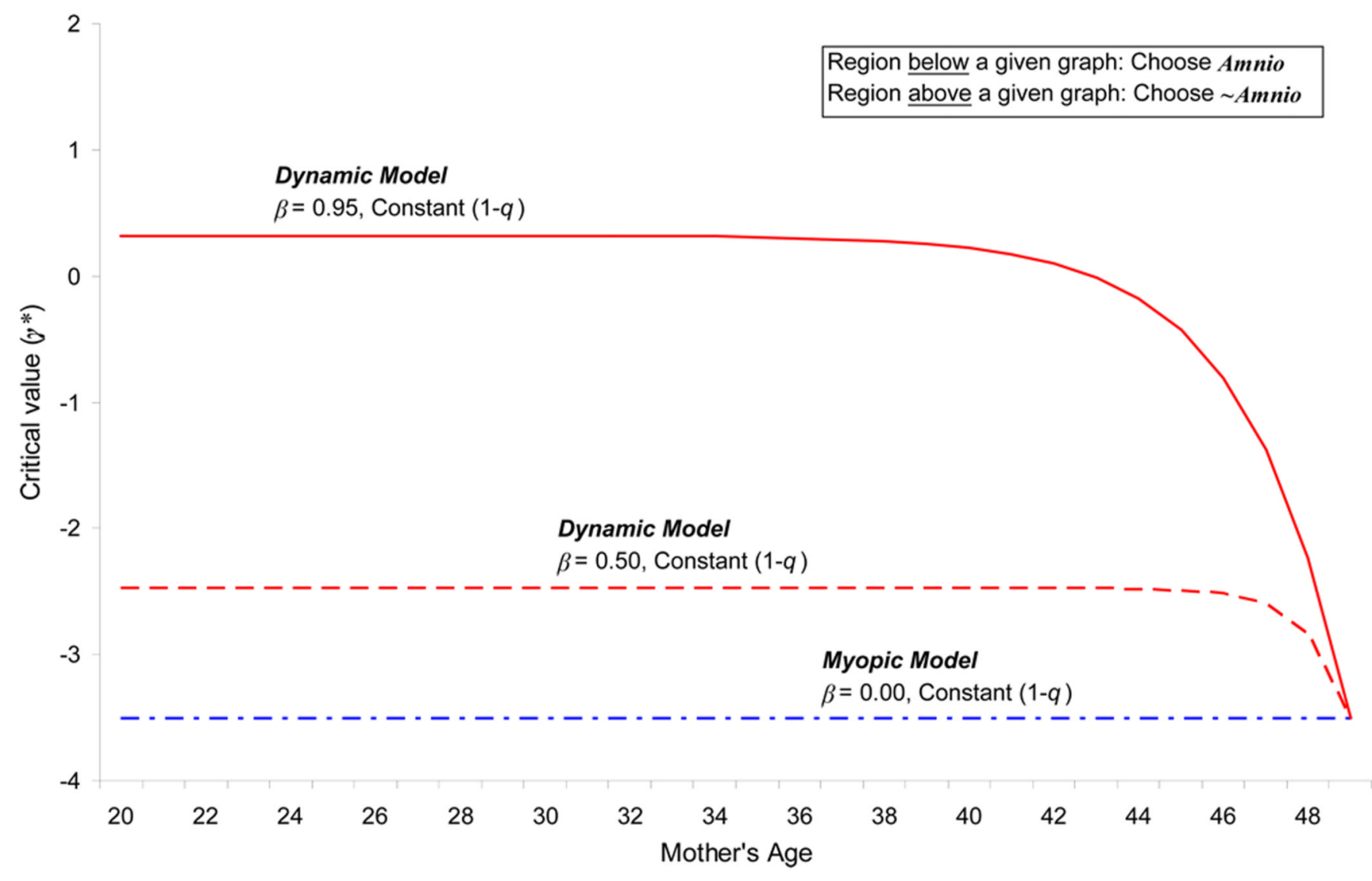

Fig. 8.

Simulations for alternative models with age-invariant probability of Down syndrome $(1-q)$ [Other assumptions: Prob. of pregnancy $(p)=0.3$; Prob. of procedure-induced miscarriage $(m)=0.0067 ;$ No costs of abortion or miscarriage $(\alpha=0 \& \mu=0)]$ 


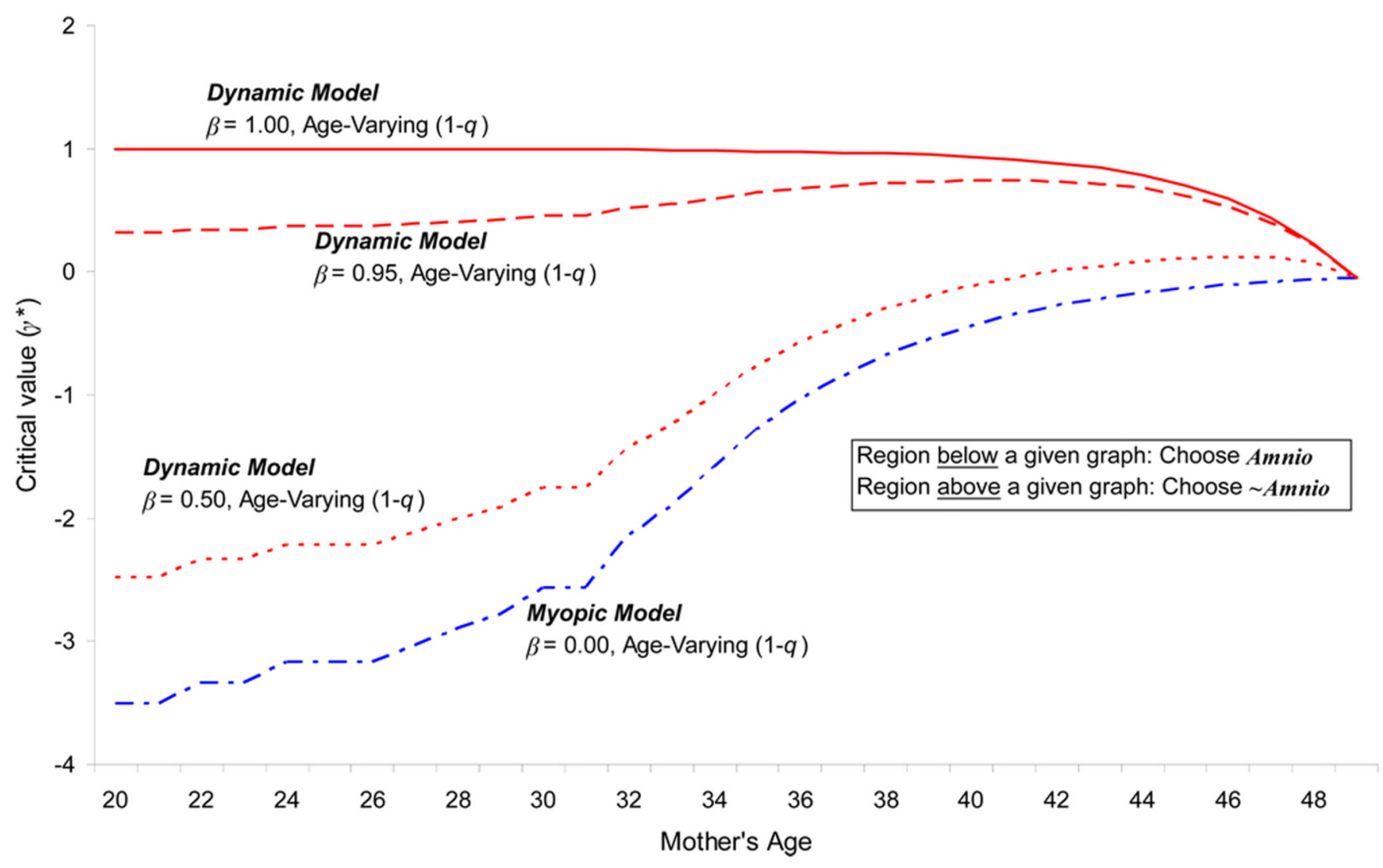

Fig. 9.

Simulations for alternative models with age-varying probability of Down syndrome $(1-q)$ [Other assumptions: Prob. of pregnancy $(p)=0.3$; Prob. of procedure-induced miscarriage $(m)=0.0067 ;$ No costs of abortion or miscarriage $(\alpha=0 \& \mu=0)]$ 


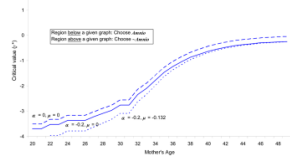

Fig. 10.

Simulations of Myopic model with alternative costs of abortion $(\alpha)$ and miscarriage $(\mu)$ [Other assumptions: Prob. of pregnancy $(p)=0.3$; Prob. of procedure-induced miscarriage $(m)=0.0067$; Age-varying prob. of Down syndrome $(1-q)]$ 


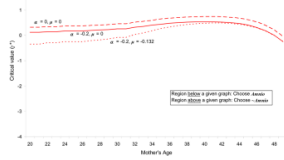

Fig. 11.

Simulations of Dynamic model with alternative costs of abortion $(\alpha)$ and miscarriage $(\mu)$ [Other assumptions: Prob. of pregnancy $(p)=0.3$; Prob. of procedure-induced miscarriage $(m)=0.0067$; Age-varying prob. of Down syndrome $(1-q)]$ 


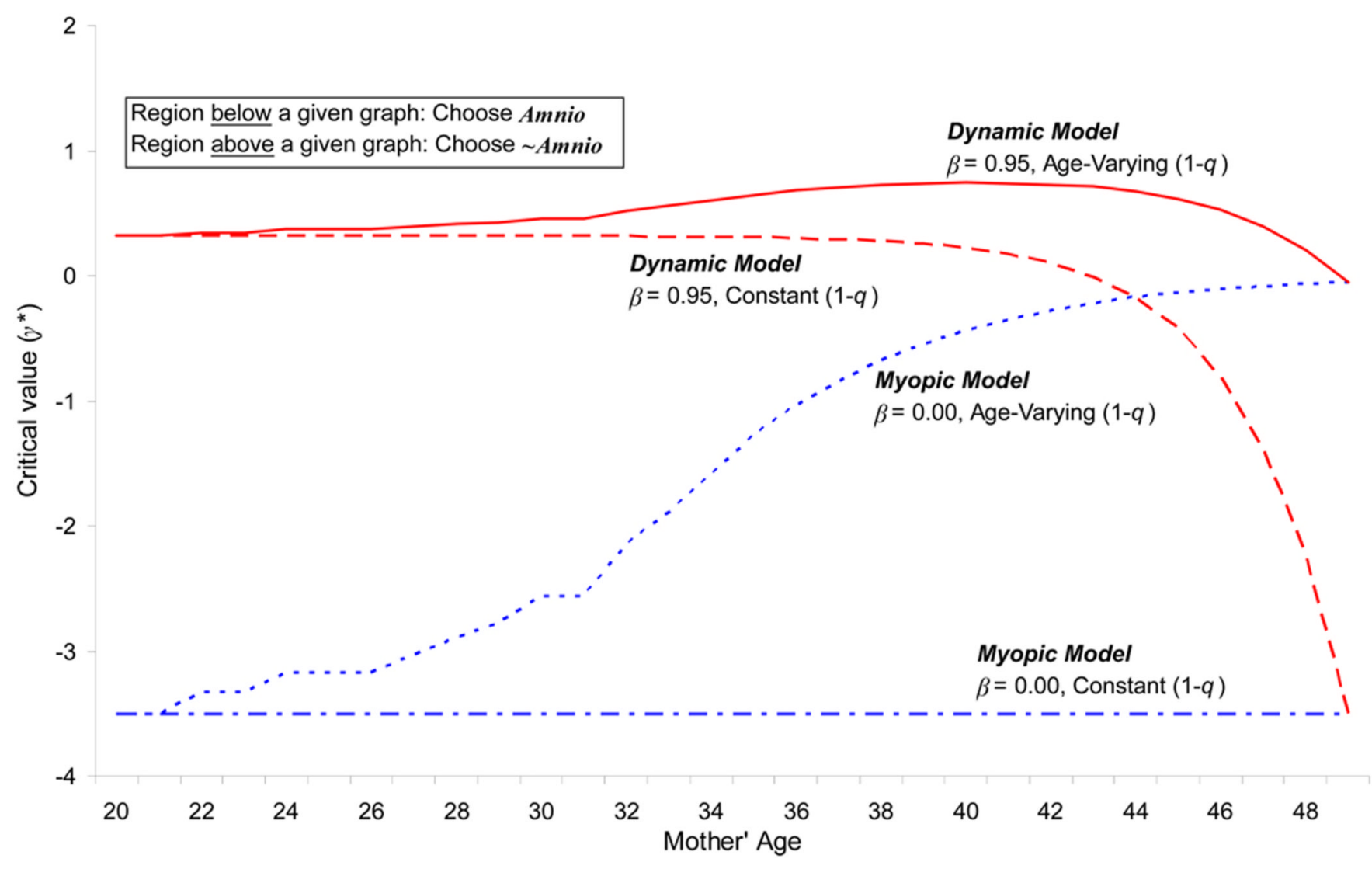

Fig. 12.

Simulations of Dynamic and Myopic models for alternative discount rates $(\beta)$ and probabilities of Down syndrome $(1-q)$ [Other assumptions: Prob. of pregnancy $(p)=0.3$; Prob. of procedure-induced miscarriage $(m)=0.0067$; No costs of abortion $(\alpha=0)$ or miscarriage $(\mu=0)$ ] 